\title{
Vacuum chamber for sample attachment in atomic force microscopy
}

\author{
Constant A. J. Putman, Kees O. van der Werf, Geeske van Oort, Bart G. de Grooth, \\ Niek F. van Hulst, and Jan Greve \\ Department of Applied Physics, University of Twente, P. O. Box 217, 7500 AE Enschede, The \\ Netherlands
}

(Received 3 February 1992; accepted for publication 10 April 1992)

\begin{abstract}
A small ring-shaped vacuum chamber has been constructed and connected to the piezotube used for scanning samples in the atomic force microscope (AFM). Samples made up of any material, up to $50 \mathrm{~mm}$ in diameter, can be firmly attached onto the piezotube without causing damage to the sample. A $50-\ell$ beer container forms a bufer between vacuum pump and chamber. With this supply of vacuum, the AFM can be operated for a 4-8 h period without turning on the vacuum pump again. Samples can be changed within $30 \mathrm{~s}$. The scan frequency when using microscope slides is limited to $40 \mathrm{~Hz}$ due to resonance effects of the microscope slides.
\end{abstract}

At the heart of the atomic force microscope (AFM) is the stylus that tracks the surface of the sample by raster scanning the sample underneath the stylus with an $x y z$ piezotube. Forces acting between the tip of the stylus and the surface deflect the cantilever. The introduction of integrated tip-cantilever sensors manufactured by microfabrication techniques ${ }^{1}$ has greatly increased the use of the AFM as a reliable research tool.

Another important aspect that contributes to good, reliable, and easy operation of the AFM is the way in which the sample is attached to the piezotube. While scanning the sample, it should stay firmly attached to the piezotube, even on a nanometer scale. In Ref. 2, a spring-loaded clip is used in the scanning tunneling microscope (STM) and a small magnet in the case of an AFM. These same techniques are also used in the commercially available Nanoscope. ${ }^{3}$ In the AFM the sample (carrier) is glued onto a small steel disk, which then is positioned on the magnet. In another commercially available $\mathrm{AFM}^{4}$ a small stub is used that can be fixed to the piezotube with a screw. To this stub the sample then can be applied using double-sticky tape or glue.

In our home-built $\mathrm{AFM}^{5}$ none of the above-mentioned sample attachment techniques have been used for different reasons. The most important being the fact that the AFM features an integrated inverted optical microscope. In this setup a lens is positioned inside the piezotube and an optical image of the (biological) specimen in back reflection or fluorescence is obtained. Thus no technique can be used that blocks the field of view of the lens. At the first stage, this problem was solved by attaching a steel cup, with a 10-mm centered hole in the bottom, upside down onto the piezotube. ${ }^{5}$ The microscope slide with the sample was then glued to the steel cup. Over a longer period of time, however, loosening and tightening the steel cup resulted in a degradation of the $\mathrm{MACOR}^{6}$ ring attached to the piezotube.

Here we present a new way of attaching samples to the piezotube. A ring-shaped vacuum chamber was constructed that is capable of holding any object, glass or metal, up to $50 \mathrm{~mm}$ in diameter.

When designing a new sample attachment device there are some conditions to be fulfilled. First, the sample should be attached to the piezotube as tight as possible: no movement on nanometer scale. Second, changing the sample should be easy and not time-consuming. Third, the device should work independent of the material of which the sample or sample substrate is made of - glass or metal.

In Fig. 1 a drawing and photograph of a vacuum chamber connected to the piezotube is shown. In order to obtain an unobstructed field of view for the lens, the vacuum chamber is ring shaped. When having no integrated inverted optical microscope, a disk-shaped vacuum chamber is the most suitable layout. The chamber is made of brass and weighs less than $15 \mathrm{~g}$. The lit of the chamber has 24 holes with a diameter of $0.75 \mathrm{~mm}$. A vacuum pump is used to evacuate the system from air and a $50-\ell$ beer container is hooked up in between the vacuum chamber and the pump. At first this was done to eliminate the pulsation on the AFM signal due to pump operation. However, it turned out that with this supply of vacuum the AFM can be operated for a period of $4-8 \mathrm{~h}$, without a need to turn on the vacuum pump again.

Having a pressure difference of $1 \mathrm{~atm}$ and a standard glass microscope slide (dimensions: $75 \times 25 \times 1 \mathrm{~mm}^{3}$, weight: $5 \mathrm{~g}$ ) the normal force is $1.1 \mathrm{~N}$. The experimental value is $2.2 \mathrm{~N}$. The difference between these two values is probably due to the conical shape of the holes and the increased area between glass slide and brass lit during lifting of the slide. The friction force between the glass microscope slide and the brass lit is directly related to the normal force. The friction coefficient between glass and polishcd brass was experimentally determined to be 0.4 . In order to have good contact and subsequently friction between lit and sample, the bottom side of the sample (carrier) should be flat. The force that is needed to move the glass slide in the horizontal direction is $0.88 \mathrm{~N}$. The experimental value is $0.9 \mathrm{~N}$. Thus, the maximum acceleration which the microscope slide can withstand without slipping is $1.8 \times 10^{2}$ $\mathrm{m} / \mathrm{s}^{2}$. The maximum frequency of a sinewave with a $10-\mu \mathrm{m}$ amplitude applied to the piezotube, before the glass slide starts slipping, can be calculated to be $6.7 \times 10^{2} \mathrm{~Hz}$. The experimental value is $5.8 \times 10^{2} \mathrm{~Hz}$. In practice the piezo is not scanned using a sine wave but with a voltage having a 

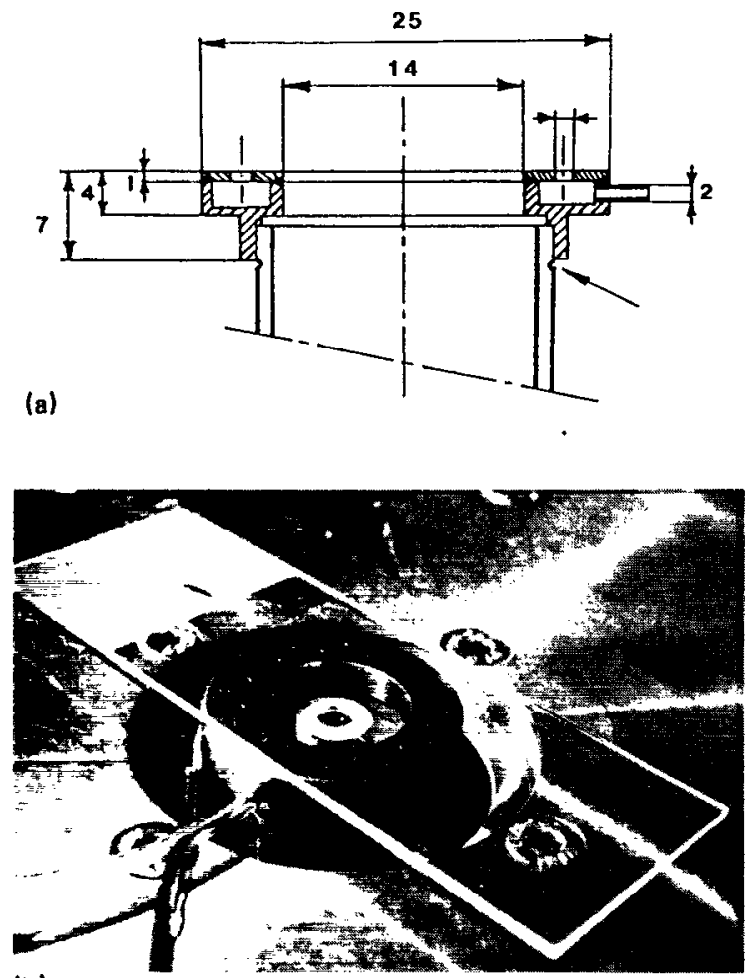

(b)

FIG. 1. Vacuum chamber attached to the piezotube. (a) All the dimensions are in $\mathrm{mm}$. In order to prevent electrical contact between the piezotube and the vacuum chamber, the $x$ and $y$ electrodes are cut close to the top of the piezo (arrow). (b) The chamber has 24 holes with a diameter of $0.75 \mathrm{~mm}$. The objective is instantly in focus when a new sample on a microscope slide is inserted. The electrical lead is for grounding and the tubing is connected with the vacuum pump.

triangular profile. Also in this case the maximum scan frequency is $5.8 \times 10^{2} \mathrm{~Hz}$. One would expect slipping at much lower frequencics because of the high acceleration forces at the turning points (in theory the acceleration has an infinite value). Due to the limited bandwidth of the highvoltage amplifier and the response of the piezotube, however, the turning points get rounded off and thus the acceleration has a finite value.
For standard microscope slides another phenomenon limits the scan frequency. At a scan frequency of $40 \mathrm{~Hz}$ the microscope slides start resonating with a frequency in the range of $250-1000 \mathrm{~Hz}$. In practice AFM images of (biological) samples are obtained at scan frequencies ranging from a few $\mathrm{Hz}$ to $20 \mathrm{~Hz}$. So this resonance effect does not limit the operation of the AFM.

Samples can be changed within $30 \mathrm{~s}$. Samples up to 50 $\mathrm{mm}$ in diameter, such as silicon wafers, can be investigated in this AFM without causing damage to the sample. Microscope slides do not have to be cut as is the case when using the commercially available AFMs ${ }^{3,4}$ and they can be transferred back and forth between the AFM and any other microscope. Since microscope slides all have a standard thickness of $1 \mathrm{~mm}$, the integrated optical microscope is instantly in focus when a new sample is inserted.

With this design the conditions, which were stated at the beginning of this section, are fulfilled. Within the limits set by the lateral resolution of the AFM, we noticed no slipping of the sample between subsequent scans. This indicates that there is no movement of the sample on a nanometer scale. Furthermore, any sample with a flat bottom side can be held down by the vacuum chamber and the changing of the samples is easy and not time consuming.

In conclusion, the introduction of a vacuum chamber for sample attachment yields a more user-friendly operation of the atomic force microscope.

We thank Klaas Smit for machining the vacuum chamber. This research was supported by the Netherlands Organization for Scientific Research NWO.

${ }^{1}$ T. R. Albrecht, S. Akamine, T. E. Carver, and C. F. Quatc, J. Vac. Sci. Technol. A 8, 3386 (1990).

${ }^{2}$ P. K. Hansma, V. B. Elings, O. Marti, and C. E. Bracker, Science 242, 209 (1988).

${ }^{3}$ Digital Instruments, Santa Barbara, California, USA.

${ }^{4}$ Park Scientific Instruments, Sunnyvale, California, USA.

${ }^{5}$ C. A. J. Putman, K. O. Van der Werf, B. G. De Grooth, N. F. Van Hulst, F. B. Segerink, and J. Greve, Rev. Sci. Instrum. 63, 1914 (1992).

${ }^{6} \mathrm{MACOR}$ is a ceramic material and is being used as an electrical insulator. 